CRYSTALLOGRAPHIC COMMUNICATIONS

ISSN 2056-9890

Received 28 November 2019

Accepted 17 February 2020

Edited by L. Van Meervelt, Katholieke Universiteit Leuven, Belgium

Keywords: co-crystallized molecules; crystal structure; tetraiodoethylene; azaphenanthrenes; luminescence.

CCDC references: 1899078; 1899077

Supporting information: this article has supporting information at journals.iucr.org/e

\section{Structural and luminescent properties of co-crystals of tetraiodoethylene with two azaphenanthrenes}

\author{
Yu-Jin Cui, ${ }^{a}$ Feng Su ${ }^{a}$ and Wei-Jun Jin ${ }^{b *}$
}

\begin{abstract}
a'Department of Chemistry, Changzhi University, Changzhi 046011, People's Republic of China, and ${ }^{\mathbf{b}}$ Key Laboratory Chemical Biology and Molecular Engineering, Education Ministry, People's Republic of China. *Correspondence e-mail: 250951251@qq.com
\end{abstract}

Two new co-crystals, tetraiodoethylene-phenanthridine $(1 / 2), 0.5 \mathrm{C}_{2} \mathrm{I}_{4} \cdot \mathrm{C}_{13} \mathrm{H}_{9} \mathrm{~N}$ (1) and tetraiodoethylene-benzo[f]quinoline (1/2), $0.5 \mathrm{C}_{2} \mathrm{I}_{4} \cdot \mathrm{C}_{13} \mathrm{H}_{9} \mathrm{~N}(2)$, were obtained from tetraiodoethylene and azaphenanthrenes, and characterized by IR and fluorescence spectroscopy, elemental analysis and X-ray crystallography. In the crystal structures, $\mathrm{C}-\mathrm{I} \cdots \pi$ and $\mathrm{C}-\mathrm{I} \cdots \mathrm{N}$ halogen bonds link the independent molecules into one-dimensional chains and two-dimensional networks with subloops. In addition, the planar azaphenanthrenes lend themselves to $\pi-\pi$ stacking and $\mathrm{C}-\mathrm{H} \cdots \pi$ interactions, leading to a diversity of supramolecular three-dimensional structural motifs being formed by these interactions. Luminescence studies show that co-crystals $\mathbf{1}$ and $\mathbf{2}$ exhibit distinctly different luminescence properties in the solid state at room temperature.

\section{Chemical context}

A halogen bond is an attractive non-covalent interaction between an electrophilic region in a covalently bonded halogen atom and a Lewis base. Halogen bonding (XB) is a powerful tool to assemble supramolecular materials and to promote chemical or biological molecular recognition (Desiraju et al., 2013; Cavallo et al., 2016; Gilday et al., 2015; Wang et al., 2016). Over the past few years, XB has been used successfully to assemble luminescent co-crystals (Liu et al., 2017a; d'Agostino et al., 2015; Ventura et al., 2014; Bolton et al., 2011). XB can play multiple roles in co-crystals, for example, as cement to assemble XB donors and acceptors together (Metrangolo et al., 2005), and, importantly, as a heavy-atom source to enhance phosphorescence or delayed fluorescence by efficient spin-orbital coupling (Gao et al., 2012). Phosphorescence or delayed fluorescence materials are very popular for preparing light devices because of the higher internal quantum efficiency of triplet excitons (Brown et al., 1993; Baldo et al., 1999).

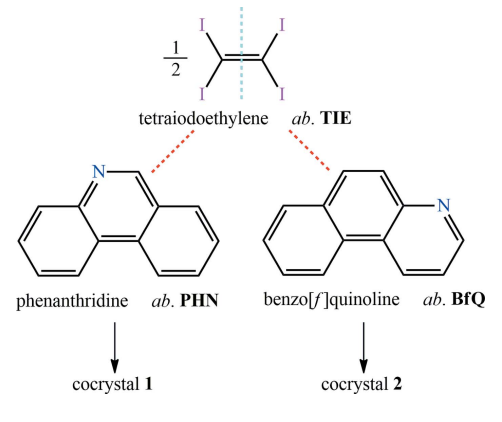

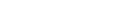


(a)
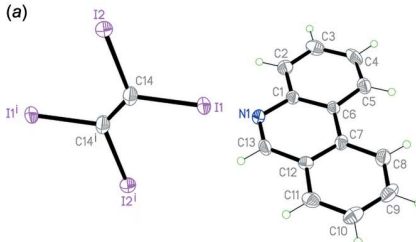

(b)
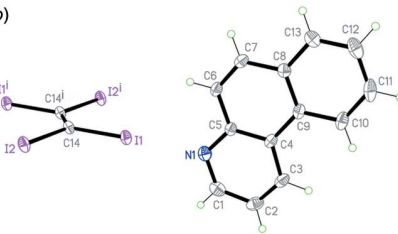

Figure 1

The molecular structures of co-crystals $\mathbf{1}$ and $\mathbf{2}$, showing the atomlabelling scheme and displacement ellipsoids at the $30 \%$ probability level [Symmetry codes: (i) $-x+\frac{3}{2},-y+\frac{3}{2},-z+1$ for co-crystal 1; (i) $-x,-y+1$, $-z+2$ for co-crystal 2].

Nitrogen heteroaromatic rings are a common type of luminescence or luminescent precursor materials. However, in general, it is difficult to use them to generate phosphorescence or delayed fluorescence. Haloperfluorobenzenes, as XB donors, have been used in attempts to assemble luminescence co-crystals with azaphenanthrenes (Gao et al., 2017; Wang et al., 2014, 2016; Wang \& Jin, 2017; Liu et al., 2017b). We report herein the use of tetraiodoethylene (TIE) as a new XB donor in the assembly of co-crystals with two different azaphenanthrenes, namely phenanthridine (PHN) and benzo[f]quinoline (BfQ), which is expected to tune their luminescence behaviour via a change of the co-crystal structures. Single crystal X-ray diffraction (XRD) data reveal that the two cocrystals of TIE with PHN and BfQ reported here have interesting structural properties and exhibit different luminescence behaviour from previous reports. TIE as a quadridentate XB donor allows the formation of three-dimensional halogenbonded networks with XB acceptors, PHN and BfQ. Using the conventional solution-based method, yellow co-crystals suitable for XRD measurement were obtained. The crystal structures of the co-crystals are mainly constructed by $\mathrm{C}-$ $\mathrm{I} \cdots \pi$ and $\mathrm{C}-\mathrm{I} \cdots \mathrm{N}$ halogen bonds. Other multiple intermolecular interactions, such as $\pi-\pi$ stacking, $\mathrm{C}-\mathrm{H} \cdots \pi, \mathrm{C}-$ $\mathrm{H} \cdots \mathrm{I}$ as well as $\mathrm{C}-\mathrm{H} \cdots \mathrm{H}-\mathrm{C}$ interactions, are also observed in the co-crystals.
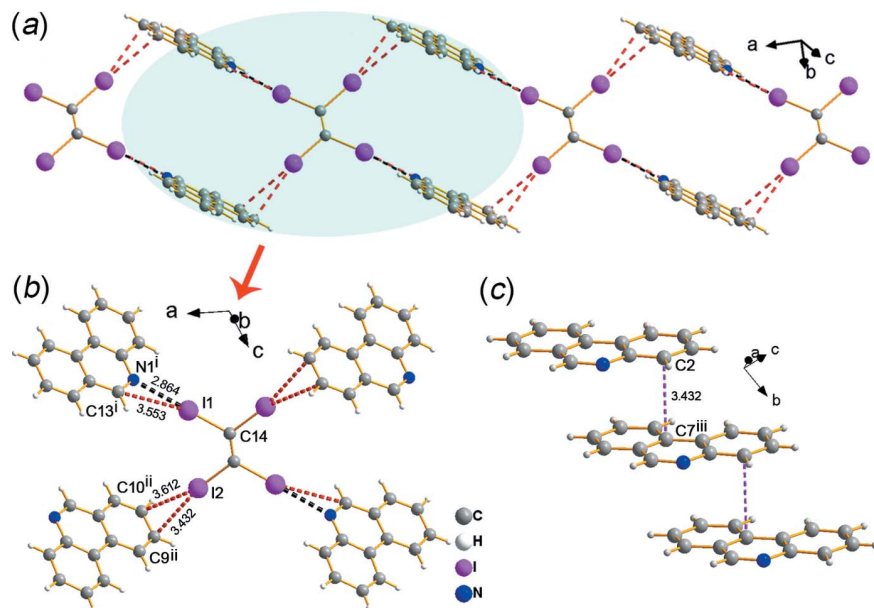

(c)

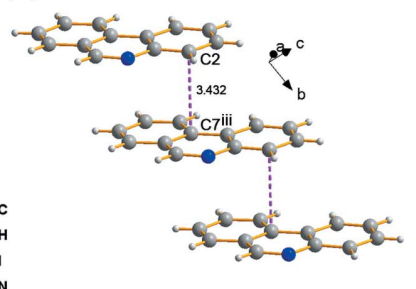

Figure 2

Crystal packing of 1. (a) The two-dimensional network structure formed by $\mathrm{C}-\mathrm{I} \cdots \mathrm{N}, \mathrm{C}-\mathrm{I} \cdots \mathrm{C}$ and $\mathrm{C}-\mathrm{I} \cdots \pi$ halogen bonds. $(b)$ The structural motif extracted from the two-dimensional network. (c) The $\pi-\pi$ stacking interactions extracted from the three-dimensional structure.

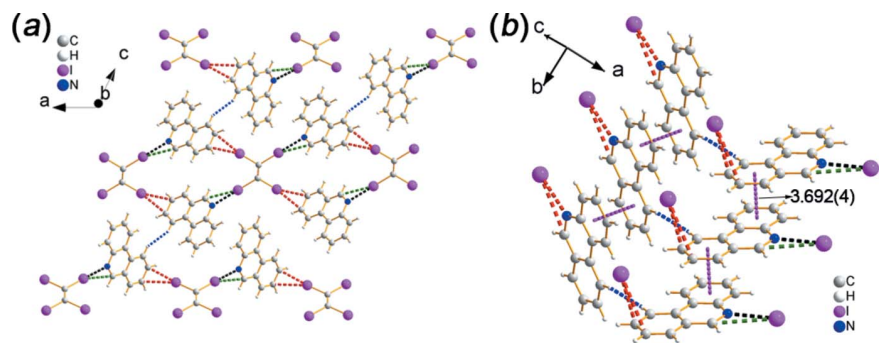

Figure 3

Crystal packing of 1. (a) The two-dimensional network extends along the $a$ - and $c$-axis directions, and two directions connect by $\mathrm{C}-\mathrm{H} \cdots \mathrm{H}-\mathrm{C}$ interactions. (b) The two-dimensional networks connected by $\pi-\pi$ stacking and $\mathrm{C}-\mathrm{H} \cdots \mathrm{H}-\mathrm{C}$ interactions to form a three-dimensional structure.

\section{Structural commentary}

The asymmetric units of co-crystals $\mathbf{1}$ and $\mathbf{2}$ each comprise one half TIE molecule lying about an inversion centre and one $\mathrm{PHN}$ or BfQ molecule in a general position, hence the cocrystals have a 1:2 stoichiometry (Fig. 1). Co-crystal 1 crystallizes in the monoclinic space group $C 2 / c$ while $\mathbf{2}$ crystallizes in the triclinic space group $P \overline{1}$.

\section{Supramolecular features}

In the crystal of $\mathbf{1}, \mathrm{C}-\mathrm{I} \cdots \mathrm{N}, \mathrm{C}-\mathrm{I} \cdots \mathrm{C}$ and $\mathrm{C}-\mathrm{I} \cdots \pi$ halogen bonds lead to the formation of a two-dimensional network structure in which the rectangular motif has a $D \cdots 2 A \cdots D \cdots 2 A$ arrangement, as shown in Fig. $2 a$. The I1 atom of the TIE molecule interacts with the N1 atom of a PHN molecule, forming a $\mathrm{C} 1-\mathrm{I} 1 \cdots \mathrm{N} 1$ halogen bond (Fig. $2 b$ ). The I1 $\cdots \mathrm{N} 1^{\mathrm{i}}$ distance is 2.864 (7) $\AA$ and the corresponding C14I1 $\cdots \mathrm{N} 1^{\mathrm{i}}$ angle is $172.8(2)^{\circ}$ [symmetry code: (i) $x, y+1, z$ ]. The strong $\mathrm{C} 14-\mathrm{I} 1 \cdots \mathrm{N} 1$ halogen bond results in a $\mathrm{I} 1 \cdots \mathrm{C} 13^{\mathrm{i}}$ distance [3.553 (8) $\AA$ ] shorter than the sum of the van der Waals radii, which indicates a $\mathrm{C} 1-\mathrm{I} 1 \cdots \mathrm{C} 13$ halogen inter-
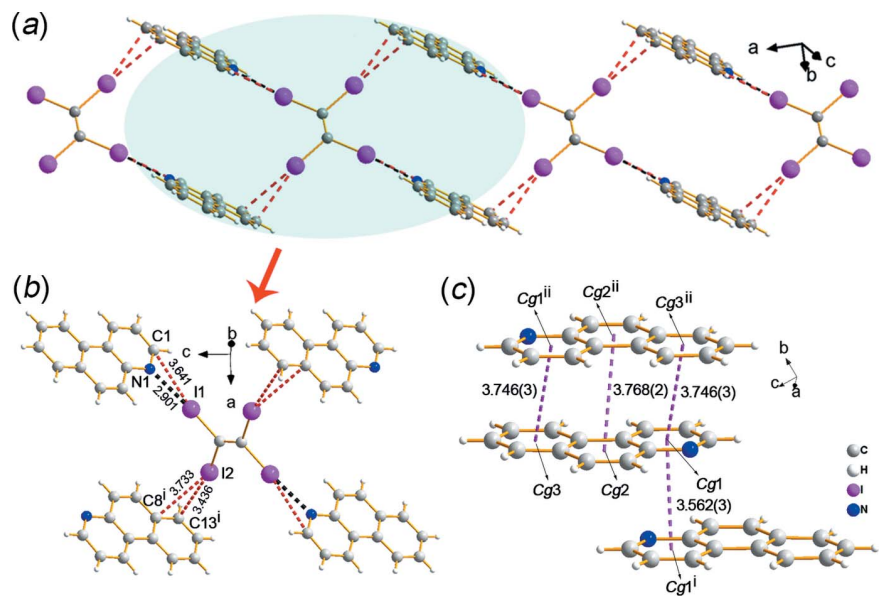

Figure 4

Crystal packing of 2. (a) The two-dimensional network structure formed by $\mathrm{C}-\mathrm{I} \cdots \mathrm{N}$ and $\mathrm{C}-\mathrm{I} \cdots \pi$ halogen bonds. (b) The structural motif of the two-dimensional network. (c) The $\pi-\pi$ stacking interactions in the threedimensional structure. 
Table 1

Hydrogen-bond geometry $\left(\AA{ }^{\circ}\right)$ for $(2)$.

\begin{tabular}{lllll}
\hline$D-\mathrm{H} \cdots A$ & $D-\mathrm{H}$ & $\mathrm{H} \cdots A$ & $D \cdots A$ & $D-\mathrm{H} \cdots A$ \\
\hline $\mathrm{C} 1-\mathrm{H} 1 \cdots \mathrm{I} 2^{\mathrm{i}}$ & 0.93 & 3.16 & $4.019(5)$ & 155 \\
$\mathrm{C} 6-\mathrm{H} 6 \cdots \mathrm{I} 1$ & 0.93 & 3.31 & $3.945(4)$ & 127 \\
\hline
\end{tabular}

Symmetry code: (i) $x+1, y, z$.

action. In addition, the $\mathrm{C} 14-\mathrm{I} 2 \cdots \mathrm{C} 9^{\mathrm{ii}} / \mathrm{C} 10^{\mathrm{ii}} \mathrm{C}-\mathrm{I} \cdots \pi$ separations [Fig. $2 b$; symmetry code: (ii) $\frac{1}{2}-x, \frac{1}{2}-y, z$ ] are 3.432 (9) and 3.612 (8) $\AA$, and the corresponding bond angles are $165.6(2)$ and $156.7(2)^{\circ}$, respectively. Furthermore, $\pi-\pi$ stacking [Fig. $2 c ; C g 1 \cdots C g 2^{\mathrm{iii}}=3.692(4) \AA, C g 3 \cdots C g 2^{\mathrm{iii}}=$ 3.626 (4) $\AA$; $C g 1, C g 2$ and $C g 3$ are the centroids of rings $\mathrm{C} 1-$ $\mathrm{C} 6, \mathrm{C} 7-\mathrm{C} 12$ and $\mathrm{N} 1 / \mathrm{C} 1 / \mathrm{C} 6 / \mathrm{C} 7 / \mathrm{C} 12 / \mathrm{C} 13$, respectively; symmetry code: (iii) $x,-1+y, z]$ and $\mathrm{C}-\mathrm{H} \cdots \mathrm{H}-\mathrm{C}$ interactions between two adjacent PHN molecules contribute to the extension of the two-dimensional network into a threedimensional supramolecular structure (Fig. 3).

The two-dimensional network of co-crystal $\mathbf{2}$ is similar to that of $\mathbf{1}$, as shown in Fig. $4 a$. Both of them are constructed by the same halogen-bonded synthon, i.e., $\mathrm{C}-\mathrm{I} \cdots \mathrm{N}, \mathrm{C}-\mathrm{I} \cdots \mathrm{C}$ and $\mathrm{C}-\mathrm{I} \cdots \pi$ halogen bonds, but the bonding characteristics are slightly different. In general, the distances of the $\mathrm{C}-\mathrm{I} \cdots \mathrm{N}$, $\mathrm{C}-\mathrm{I} \cdots \mathrm{C}$ and $\mathrm{C}-\mathrm{I} \cdots \pi$ interactions $[\mathrm{I} 1 \cdots \mathrm{N} 1=2.901(4)$, $\mathrm{I} 1 \cdots \mathrm{C} 1=3.641(5), \mathrm{I} 2 \cdots \mathrm{C} 13(-x, 1-y, 1-z)=3.436(5)$ and $\mathrm{I} 2 \cdots \mathrm{C} 8(-x, 1-y, 1-z)=3.733$ (4) $\AA$, respectively] in cocrystal 2 are all a little longer $(0.004-0.121 \AA)$ than in $\mathbf{1}$ (Fig. 4b). In addition, the two-dimensional network (Fig. 5) is extended to a three-dimensional supramolecular structure by $\pi-\pi$ stacking (Fig. $4 c$ and 5; $C g 1 \cdots C g 1^{\mathrm{i}}=3.562(3) \AA$, $C g 1 \cdots C g 2^{\mathrm{ii}}=3.963(2) \AA, \quad C g 1 \cdots C g 3^{\mathrm{ii}}=3.746(3) \AA$,

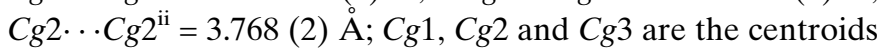
of rings $\mathrm{N} 1 / \mathrm{C} 1-\mathrm{C} 5, \mathrm{C} 4-\mathrm{C} 9$ and $\mathrm{C} 8-\mathrm{C} 13$, respectively; symmetry codes: (i) $1-x, 1-y, 1-z$; (ii) $1-x,-y, 1-z$ ] and $\mathrm{C}-\mathrm{H} \cdots \mathrm{I}$ hydrogen bonds (Table 1 ).

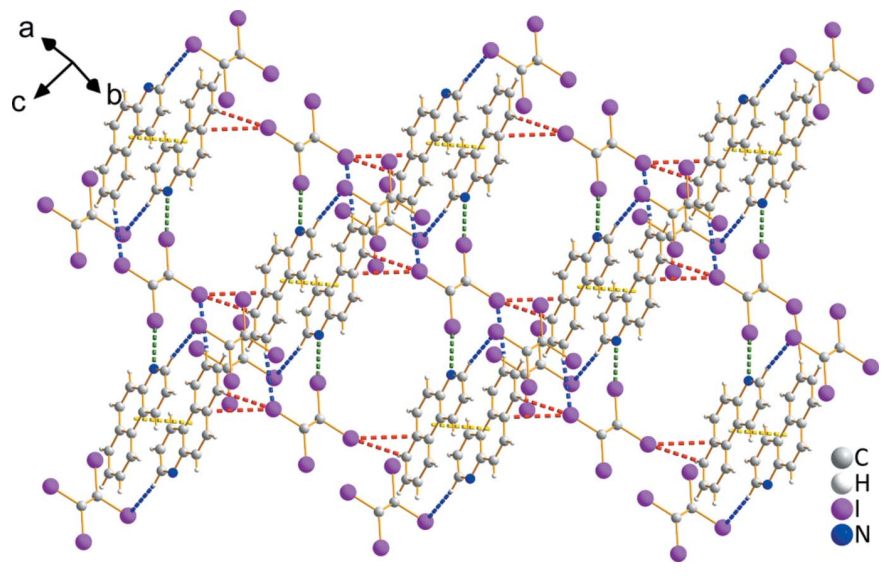

Figure 5

Crystal packing of $\mathbf{2}$. The two-dimensional network extends along two directions, by $\mathrm{C}-\mathrm{I} 2 \cdots \mathrm{H} 7$ interactions and $\pi-\pi$ stacking in one direction, and by $\mathrm{C}-\mathrm{I} 2 \cdots \mathrm{H} 1$ interactions and $\pi-\pi$ stacking in the other.
Table 2

Phosphorescent characteristics of co-crystals at room temperature.

\begin{tabular}{llll}
\hline & & TIE-PHN & TIE-BfQ \\
\hline $\begin{array}{l}\text { Total luminescent } \\
\text { spectra }\end{array}$ & $\lambda_{\text {ex }} / \mathrm{nm}$ & 300 & 300 \\
& $\lambda_{\text {em }} / \mathrm{nm}$ & $375,484,578$ & $368,438,452,480$ \\
& $\tau_{\text {average }} / \mathrm{ns}$ & 11.49 & 9.29 \\
DF and phosphorescent & $\lambda_{\text {ex }} / \mathrm{nm}$ & 330 & 330 \\
$\quad$ spectra & $\lambda_{\text {em }} / \mathrm{nm}$ & $375,489,600$ & $430,489,596,654$ \\
& $\tau_{\text {average }} / \mu \mathrm{s}$ & 4.36 & 6.45 \\
\hline
\end{tabular}

\section{Powder X-ray diffraction pattern}

The powder X-ray diffraction (PXRD) experiments were carried out for the title co-crystals using a Bruker D8ADVANCE X-ray diffractometer $(\mathrm{Cu} K \alpha, \lambda=1.5418 \AA)$ in the $2 \theta$ range of 5 to $50^{\circ}$. As shown in Fig. 6 , the experimental patterns for $\mathbf{1}$ and $\mathbf{2}$ match well with the spectra simulated from the XRD data, which confirms the purity of $\mathbf{1}$ and $\mathbf{2}$.

\section{Luminescence behavior of co-crystals 1 and 2}

As shown in Fig. 7, the two co-crystals fluoresce with some vibrational fine structure (see also spectroscopic data in Table 2). The two co-crystals also show delayed fluorescence (Fig. 8). For both co-crystals, the emission bands in the region of $450-480 \mathrm{~nm}$ should be relative to the $\pi-\pi$ stacking patterns. Luminescence from the excimer is possible because of the close $\pi-\pi$ stacking distances as shown in Figs. 2-5, besides luminescence from a monomer. Furthermore, TIE-PHN and TIE-BfQ produce weak phosphorescence. The strong XB interaction between the iodine atoms of TIE and the nonbonding orbitals of the azaphenanthrene $\mathrm{N}$ atoms should cause the energy of the lowest ${ }^{1}\left(n, \pi^{*}\right)$ state to drop below that of the ${ }^{3}\left(\pi, \pi^{*}\right)$ state. It is supposed that for the singlet states the $0-0$ transition of emitters in co-crystals is localized at $375 \mathrm{~nm}$ and $450 \mathrm{~nm}$, respectively, and for triplet states the $0-0$ transition is at about $600 \mathrm{~nm}$. The energy gap between $S_{1}$ and $T_{1}$ is largely greater than $20 \mathrm{~kJ} \mathrm{~mol}^{-1}$, so the delayed fluorescence most likely originates from the triplet-triplet annihilation process, named $P$-type delayed fluorescence (P-DF). Both delayed fluorescence and phosphorescence are relative to triplet states, so they should be significant for
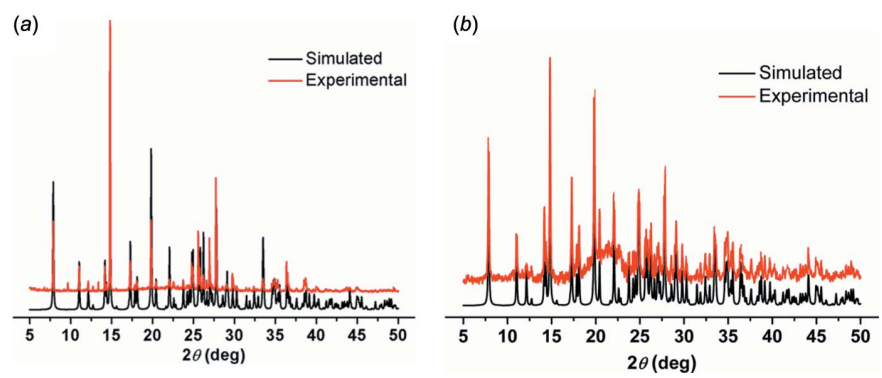

Figure 6

Powder X-ray diffraction pattern of co-crystals $(a) \mathbf{1}$ and (b) $\mathbf{2}$. 
Table 3

Experimental details.

1

Crystal data

Chemical formula

$M_{\mathrm{r}}$

Crystal system, space group

Temperature (K)

$a, b, c(\AA)$

$\alpha, \beta, \gamma\left({ }^{\circ}\right)$

$V\left(\AA^{3}\right)$

$Z$

Radiation type

$\mu\left(\mathrm{mm}^{-1}\right)$

Crystal size (mm)

Data collection

Diffractometer

Absorption correction

$T_{\min }, T_{\max }$

No. of measured, independent and

observed $[I>2 \sigma(I)]$ reflections

$R_{\text {int }}$

$(\sin \theta / \lambda)_{\max }\left(\AA^{-1}\right)$

Refinement

$R\left[F^{2}>2 \sigma\left(F^{2}\right)\right], w R\left(F^{2}\right), S$

No. of reflections

No. of parameters

$\mathrm{H}$-atom treatment

$\Delta \rho_{\max }, \Delta \rho_{\min }\left(\mathrm{e} \AA^{-3}\right)$
$0.5 \mathrm{C}_{2} \mathrm{I}_{4} \cdot \mathrm{C}_{13} \mathrm{H}_{9} \mathrm{~N}$

445.02

Monoclinic, $C 2 / c$

296

24.2920 (18), 4.8348 (4), 24.8761 (16)

90, $116.272(2), 90$

$2619.8(3)$

8

Mo $K \alpha$

4.78

$0.30 \times 0.25 \times 0.25$

Bruker APEXII CCD

Multi-scan (SADABS; Bruker, 2012)

$0.489,0.745$

12094, 2635, 1782

0.045

0.624

$0.043,0.078,1.03$

2635

154

$\mathrm{H}$-atom parameters constrained

$1.08,-0.62$
2

$0.5 \mathrm{C}_{2} \mathrm{I}_{4} \cdot \mathrm{C}_{13} \mathrm{H}_{9} \mathrm{~N}$

445.02

Triclinic, $P \overline{1}$

296

7.3179 (4), 8.1089 (5), 11.3252 (7)

$97.050(2), 92.059(2), 95.579(2)$

$663.02(7)$

2

Mo $K \alpha$

4.72

$0.35 \times 0.32 \times 0.30$

Bruker APEXII CCD

Multi-scan (SADABS; Bruker, 2012)

$0.638,0.745$

8567, 2701, 2099

0.026

0.625

$0.028,0.058,1.08$

2701

154

H-atom parameters constrained $0.80,-0.74$

Computer programs: APEX2 and SAINT (Bruker, 2012), SHELXS97 and SHELXTL (Sheldrick, 2008), SHELXL2018/3 (Sheldrick, 2015), (Sheldrick, 2008), DIAMOND (Brandenburg, 2005) and publCIF (Westrip, 2010).

improving the exciton emission efficiency of luminescence materials (Adachi et al., 2001).

For the luminescence decay, all singlet state decay lifetimes (11.49 ns for $\mathbf{1}$ and $9.29 \mathrm{~ns}$ for $\mathbf{2}$ ) are about $10 \mathrm{~ns}$, while the
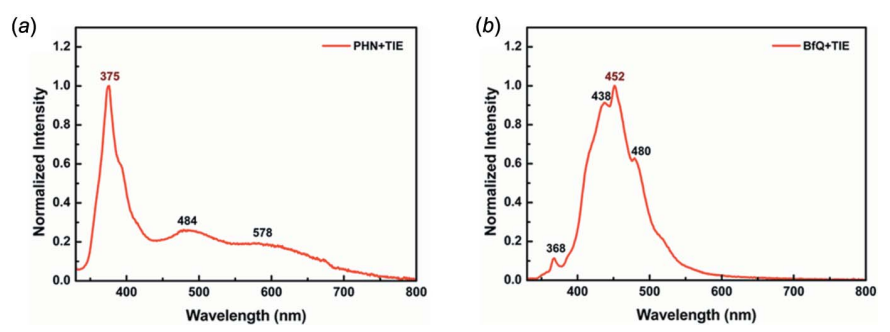

Figure 7

Total luminescence spectra of co-crystals $(a) \mathbf{1}$ and $(b) \mathbf{2}$ (excitation at $300 \mathrm{~nm}$ ) measured under fluorescence mode.
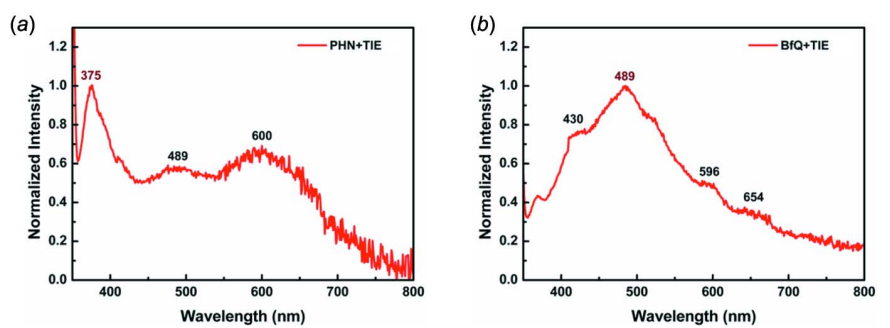

Figure 8

Luminescence spectra of co-crystals $(a) \mathbf{1}$ and $(b) \mathbf{2}$ (excitation at $330 \mathrm{~nm}$ ) measured under phosphorescence mode. delayed fluorescence lifetime ( $4.36 \mu$ s for $\mathbf{1}$ and $6.45 \mu$ s for 2$)$ is less than the $10 \mu$ s level because of the strong heavy-atom effect leading to a faster decay of the triplet state. Additionally, the phosphorescence is too weak to measure its decay lifetime. However, the phosphorescence lifetime can be estimated to be about $20 \mu$ s based on the relationship between PDF and the accompanying phosphorescence (Parker et al., 1962, 1965).

\section{Synthesis and crystallization}

$0.1 \mathrm{mmol}$ of PHN/BfQ and $0.05 \mathrm{mmol}$ of TIE were dissolved in an acetone/chloroform (2:1) mixture in a glass vial. Wellformed co-crystals $\mathbf{1}$ and $\mathbf{2}$ suitable for single-crystal X-ray diffraction (XRD) measurements were obtained by slow evaporation of the solvent at room temperature after about two weeks. Elemental analysis (\%, EA) calculated for $\mathrm{C}_{14} \mathrm{H}_{9} \mathrm{NI}_{2}$ (445.02): C 37.78, H 2.04, N 3.15. Found: C 37.54, H 2.31, N 3.26. For co-crystal 1, and C 37.85, H 2.16, N 3.04 for co-crystal 2. IR (KBr, v, $\mathrm{cm}^{-1}$ ) For 1: 3048(w), 1603(w), 1572(w), 1494(m), 1446(w), 1382(m), 1293(m), 1267(m), 1189(m), 1089(m), 948(m), 870(s), 832(s), 802(s), 749(s), $707(s), 615(m), 538(m), 487(m), 435(m)$. For 2: 3048(w), 1611(w), 1576(s), 1522(w), 1486(w), 1458(m), 1440(m), 1238(m), 1132(m), 1032(m), 953(m), 924(m), 889(s), 745(s), 714(s), 610(m), 552(m), 448(m), 423(m). 


\section{Refinement}

Crystal data, data collection and structure refinement details are summarized in Table 3 . $\mathrm{H}$ atoms attached to $\mathrm{C}$ atoms were positioned geometrically and refined as riding on their parent atoms, with $\mathrm{C}-\mathrm{H}=0.93 \AA$ and $U_{\text {iso }}(\mathrm{H})=1.2 U_{\text {eq }}(\mathrm{C})$.

\section{Funding information}

The authors are grateful for support by the Scientific and Technological Innovation Programs of Higher Education Institutions in Shanxi Province (grant No. 2019L0895) and the Undergraduate Innovation and Entrepreneurship Project of Changzhi University (award No. zz201814).

\section{References}

Adachi, C., Baldo, M. A., Thompson, M. E. \& Forrest, S. R. (2001). J. Appl. Phys. 90, 5048-5051.

Agostino, S. d', Grepioni, F., Braga, D. \& Ventura, B. (2015). Cryst. Growth Des. 15, 2039-2045.

Baldo, M. A., O'Brien, D. F., Thompson, M. E. \& Forrest, S. R. (1999). Phys. Rev. B, 60, 14422-14428.

Bolton, O., Lee, K., Kim, H. J., Lin, K. Y. \& Kim, J. (2011). Nat. Chem. 3, 205-210.

Brandenburg, K. (2005). DIAMOND. Crystal Impact GbR, Bonn, Germany.

Brown, A. R., Pichler, K., Greenham, N. C., Bradley, D. D. C., Friend, R. H. \& Holmes, A. B. (1993). Chem. Phys. Lett. 210, 61-66.

Bruker (2012). APEX2, SAINT and SADABS. Bruker AXS Inc., Madison, Wisconsin, USA.
Cavallo, G., Metrangolo, P., Milani, R., Pilati, T., Priimagi, A., Resnati, G. \& Terraneo, G. (2016). Chem. Rev. 116, 2478-2601.

Desiraju, G. R., Ho, P. S., Kloo, L., Legon, A. C., Marquardt, R., Metrangolo, P., Politzer, P., Resnati, G. \& Rissanen, K. (2013). Pure Appl. Chem. 85, 1711-1713.

Gao, H. Y., Shen, Q. J., Zhao, X. R., Yan, X. Q., Pang, X. \& Jin, W. J. (2012). J. Mater. Chem. 22, 5336-5343.

Gao, Y. J., Li, C., Liu, R. \& Jin, W. J. (2017). Spectrochim. Acta A, 173, 792-799.

Gilday, L. C., Robinson, S. W., Barendt, T. A., Langton, M. J., Mullaney, B. R. \& Beer, P. D. (2015). Chem. Rev. 115, 71187195.

Liu, R., Gao, Y. J. \& Jin, W. J. (2017a). Acta Cryst. B73, 247-254.

Liu, R., Wang, H. \& Jin, W. J. (2017b). Cryst. Growth Des. 17, 33313337.

Metrangolo, P., Neukirch, H., Pilati, T. \& Resnati, G. (2005). Acc. Chem. Res. 38, 386-395.

Parker, C. A. \& Hatchard, C. G. (1962). Proc. Roy. Soc. A, 269, 574 584.

Parker, C. A., Hatchard, C. G. \& Joyce, T. A. (1965). Nature, 205, 1282-1284.

Sheldrick, G. M. (2008). Acta Cryst. A64, 112-122.

Sheldrick, G. M. (2015). Acta Cryst. C71, 3-8.

Ventura, B., Bertocco, A., Braga, D., Catalano, L., d'Agostino, S., Grepioni, F. \& Taddei, P. (2014). J. Phys. Chem. C, 118, 1864618658.

Wang, H., Hu, R. X., Pang, X., Gao, H. Y. \& Jin, W. J. (2014). CrystEngComm, 16, 7942-7948.

Wang, H. \& Jin, W. J. (2017). Acta Cryst. B73, 210-216.

Wang, H., Wang, W. \& Jin, W. J. (2016). Chem. Rev. 116, 5072-5104.

Westrip, S. P. (2010). J. Appl. Cryst. 43, 920-925. 


\section{supporting information}

Acta Cryst. (2020). E76, 438-442 [https://doi.org/10.1107/S2056989020002182]

\section{Structural and luminescent properties of co-crystals of tetraiodoethylene with two azaphenanthrenes}

\section{Yu-Jin Cui, Feng Su and Wei-Jun Jin}

Computing details

For both structures, data collection: APEX2 (Bruker, 2012); cell refinement: SAINT (Bruker, 2012); data reduction: SAINT (Bruker, 2012); program(s) used to solve structure: SHELXS97 (Sheldrick, 2008); program(s) used to refine structure: SHELXL2018/3 (Sheldrick, 2015). Molecular graphics: SHELXTL (Sheldrick, 2008), DIAMOND (Brandenburg, 2005) for (1); SHELXTL (Sheldrick, 2008), DIAMOND (Brandenburg, 2005), for (2). For both structures, software used to prepare material for publication: publCIF (Westrip, 2010).

Tetraiodoethylene-phenanthridine (1/2) (1)

Crystal data

$0.5 \mathrm{C}_{2} \mathrm{I}_{4} \cdot \mathrm{C}_{13} \mathrm{H}_{9} \mathrm{~N}$

$M_{r}=445.02$

Monoclinic, $C 2 / c$

$a=24.2920(18) \AA$

$b=4.8348(4) \AA$

$c=24.8761(16) \AA$

$\beta=116.272(2)^{\circ}$

$V=2619.8(3) \AA^{3}$

$Z=8$

Data collection

Bruker APEXII CCD diffractometer

Radiation source: sealed tube $\varphi$ and $\omega$ scans

Absorption correction: multi-scan

(SADABS; Bruker, 2012)

$T_{\text {min }}=0.489, T_{\max }=0.745$

12094 measured reflections

Refinement

Refinement on $F^{2}$

Least-squares matrix: full

$R\left[F^{2}>2 \sigma\left(F^{2}\right)\right]=0.043$

$w R\left(F^{2}\right)=0.078$

$S=1.03$

2635 reflections

154 parameters

0 restraints
$F(000)=1648$

$D_{\mathrm{x}}=2.257 \mathrm{Mg} \mathrm{m}^{-3}$

Mo $K \alpha$ radiation, $\lambda=0.71073 \AA$

Cell parameters from 5311 reflections

$\theta=3.1-26.3^{\circ}$

$\mu=4.78 \mathrm{~mm}^{-1}$

$T=296 \mathrm{~K}$

Block, yellow

$0.30 \times 0.25 \times 0.25 \mathrm{~mm}$

2635 independent reflections

1782 reflections with $I>2 \sigma(I)$

$R_{\text {int }}=0.045$

$\theta_{\text {max }}=26.3^{\circ}, \theta_{\min }=3.1^{\circ}$

$h=-30 \rightarrow 29$

$k=-6 \rightarrow 6$

$l=-31 \rightarrow 30$

Hydrogen site location: inferred from neighbouring sites

$\mathrm{H}$-atom parameters constrained

$w=1 /\left[\sigma^{2}\left(F_{\mathrm{o}}{ }^{2}\right)+(0.0187 P)^{2}+28.9715 P\right]$

where $P=\left(F_{\mathrm{o}}^{2}+2 F_{\mathrm{c}}{ }^{2}\right) / 3$

$(\Delta / \sigma)_{\max }=0.001$

$\Delta \rho_{\max }=1.08 \mathrm{e} \AA^{-3}$

$\Delta \rho_{\min }=-0.61$ e $\AA^{-3}$ 


\section{Special details}

Geometry. All esds (except the esd in the dihedral angle between two 1.s. planes) are estimated using the full covariance matrix. The cell esds are taken into account individually in the estimation of esds in distances, angles and torsion angles; correlations between esds in cell parameters are only used when they are defined by crystal symmetry. An approximate (isotropic) treatment of cell esds is used for estimating esds involving l.s. planes.

Fractional atomic coordinates and isotropic or equivalent isotropic displacement parameters $\left(\AA^{2}\right)$

\begin{tabular}{lllll}
\hline & $x$ & $y$ & $z$ & $U_{\text {iso }} * U_{\text {eq }}$ \\
\hline I1 & $0.70990(2)$ & $0.95088(10)$ & $0.57060(2)$ & $0.04011(16)$ \\
I2 & $0.85225(2)$ & $0.96131(11)$ & $0.56611(2)$ & $0.04316(16)$ \\
N1 & $0.6417(3)$ & $0.1927(12)$ & $0.6276(2)$ & $0.0377(14)$ \\
C1 & $0.6440(3)$ & $0.1453(14)$ & $0.6836(3)$ & $0.0339(16)$ \\
C2 & $0.6849(3)$ & $-0.0548(15)$ & $0.7193(3)$ & $0.0442(18)$ \\
H2 & 0.708323 & -0.152775 & 0.704671 & $0.053^{*}$ \\
C3 & $0.6910(4)$ & $-0.1093(16)$ & $0.7755(3)$ & $0.053(2)$ \\
H3 & 0.719345 & -0.239978 & 0.799494 & $0.064^{*}$ \\
C4 & $0.6547(4)$ & $0.0319(17)$ & $0.7965(3)$ & $0.054(2)$ \\
H4 & 0.658318 & -0.006933 & 0.834537 & $0.065^{*}$ \\
C5 & $0.6141(3)$ & $0.2253(16)$ & $0.7623(3)$ & $0.0452(19)$ \\
H5 & 0.590382 & 0.318582 & 0.777344 & $0.054^{*}$ \\
C6 & $0.6070(3)$ & $0.2880(14)$ & $0.7044(3)$ & $0.0328(16)$ \\
C7 & $0.5640(3)$ & $0.4901(13)$ & $0.6650(3)$ & $0.0342(16)$ \\
C8 & $0.5240(3)$ & $0.6464(16)$ & $0.6801(3)$ & $0.0475(19)$ \\
H8 & 0.524161 & 0.622630 & 0.717264 & $0.057^{*}$ \\
C9 & $0.4853(3)$ & $0.8313(17)$ & $0.6406(4)$ & $0.054(2)$ \\
H9 & 0.459174 & 0.933001 & 0.651309 & $0.065^{*}$ \\
C10 & $0.4834(3)$ & $0.8741(16)$ & $0.5846(4)$ & $0.051(2)$ \\
H10 & 0.456128 & 1.001451 & 0.558148 & $0.061^{*}$ \\
C11 & $0.5219(3)$ & $0.7271(15)$ & $0.5691(3)$ & $0.0455(19)$ \\
H11 & 0.521208 & 0.755053 & 0.531834 & $0.055^{*}$ \\
C12 & $0.5627(3)$ & $0.5329(14)$ & $0.6091(3)$ & $0.0345(16)$ \\
C13 & $0.6035(3)$ & $0.3779(15)$ & $0.5941(3)$ & $0.0405(18)$ \\
H13 & 0.602707 & 0.412409 & 0.557015 & $0.049^{*}$ \\
C14 & $0.7603(3)$ & $0.8233(15)$ & $0.5244(3)$ & $0.0411(18)$ \\
& & & & \\
\end{tabular}

Atomic displacement parameters $\left(\AA^{2}\right)$

\begin{tabular}{lllllll}
\hline & $U^{11}$ & $U^{22}$ & $U^{33}$ & $U^{12}$ & $U^{13}$ & $U^{23}$ \\
\hline $\mathrm{I} 1$ & $0.0411(3)$ & $0.0521(3)$ & $0.0327(3)$ & $0.0057(2)$ & $0.0213(2)$ & $-0.0022(2)$ \\
$\mathrm{I} 2$ & $0.0332(3)$ & $0.0582(3)$ & $0.0364(3)$ & $-0.0048(2)$ & $0.0139(2)$ & $-0.0059(2)$ \\
$\mathrm{N} 1$ & $0.034(3)$ & $0.046(4)$ & $0.037(3)$ & $0.000(3)$ & $0.019(3)$ & $-0.004(3)$ \\
$\mathrm{C} 1$ & $0.034(4)$ & $0.036(4)$ & $0.033(4)$ & $-0.005(3)$ & $0.016(3)$ & $-0.005(3)$ \\
$\mathrm{C} 2$ & $0.042(4)$ & $0.043(4)$ & $0.046(4)$ & $0.001(4)$ & $0.018(4)$ & $0.001(4)$ \\
$\mathrm{C} 3$ & $0.060(5)$ & $0.049(5)$ & $0.041(5)$ & $0.000(4)$ & $0.013(4)$ & $0.011(4)$ \\
$\mathrm{C} 4$ & $0.067(6)$ & $0.066(6)$ & $0.027(4)$ & $-0.015(5)$ & $0.019(4)$ & $-0.001(4)$ \\
$\mathrm{C} 5$ & $0.048(5)$ & $0.053(5)$ & $0.041(4)$ & $-0.010(4)$ & $0.026(4)$ & $-0.008(4)$ \\
$\mathrm{C} 6$ & $0.031(4)$ & $0.038(4)$ & $0.030(4)$ & $-0.008(3)$ & $0.014(3)$ & $-0.007(3)$
\end{tabular}




\begin{tabular}{lllllll} 
C7 & $0.032(4)$ & $0.034(4)$ & $0.041(4)$ & $-0.006(3)$ & $0.020(3)$ & $-0.008(3)$ \\
C8 & $0.045(5)$ & $0.053(5)$ & $0.049(5)$ & $-0.002(4)$ & $0.026(4)$ & $-0.005(4)$ \\
C9 & $0.031(5)$ & $0.051(5)$ & $0.081(6)$ & $-0.003(4)$ & $0.025(4)$ & $-0.015(5)$ \\
C10 & $0.038(5)$ & $0.040(5)$ & $0.070(6)$ & $0.006(4)$ & $0.019(4)$ & $0.005(4)$ \\
C11 & $0.042(5)$ & $0.049(5)$ & $0.039(4)$ & $-0.012(4)$ & $0.012(4)$ & $-0.003(4)$ \\
C12 & $0.030(4)$ & $0.036(4)$ & $0.033(4)$ & $-0.003(3)$ & $0.010(3)$ & $-0.001(3)$ \\
C13 & $0.046(5)$ & $0.052(5)$ & $0.030(4)$ & $-0.005(4)$ & $0.023(4)$ & $-0.002(4)$ \\
C14 & $0.045(5)$ & $0.044(5)$ & $0.043(4)$ & $0.007(4)$ & $0.028(4)$ & $0.003(3)$ \\
\hline
\end{tabular}

Geometric parameters $\left(\AA,{ }^{\circ}\right)$

\begin{tabular}{|c|c|c|c|}
\hline I1-C14 & $2.108(6)$ & $\mathrm{C} 6-\mathrm{C} 7$ & $1.449(9)$ \\
\hline $\mathrm{I} 2-\mathrm{C} 14$ & $2.111(7)$ & $\mathrm{C} 7-\mathrm{C} 12$ & $1.394(9)$ \\
\hline $\mathrm{N} 1-\mathrm{C} 13$ & $1.293(8)$ & $\mathrm{C} 7-\mathrm{C} 8$ & $1.406(9)$ \\
\hline $\mathrm{N} 1-\mathrm{C} 1$ & $1.390(8)$ & $\mathrm{C} 8-\mathrm{C} 9$ & $1.353(10)$ \\
\hline $\mathrm{C} 1-\mathrm{C} 2$ & $1.389(9)$ & $\mathrm{C} 8-\mathrm{H} 8$ & 0.9300 \\
\hline $\mathrm{C} 1-\mathrm{C} 6$ & $1.400(9)$ & $\mathrm{C} 9-\mathrm{C} 10$ & $1.387(11)$ \\
\hline $\mathrm{C} 2-\mathrm{C} 3$ & $1.364(10)$ & C9-H9 & 0.9300 \\
\hline $\mathrm{C} 2-\mathrm{H} 2$ & 0.9300 & $\mathrm{C} 10-\mathrm{C} 11$ & $1.360(10)$ \\
\hline $\mathrm{C} 3-\mathrm{C} 4$ & $1.389(11)$ & $\mathrm{C} 10-\mathrm{H} 10$ & 0.9300 \\
\hline $\mathrm{C} 3-\mathrm{H} 3$ & 0.9300 & $\mathrm{C} 11-\mathrm{C} 12$ & $1.408(9)$ \\
\hline $\mathrm{C} 4-\mathrm{C} 5$ & $1.352(10)$ & $\mathrm{C} 11-\mathrm{H} 11$ & 0.9300 \\
\hline $\mathrm{C} 4-\mathrm{H} 4$ & 0.9300 & $\mathrm{C} 12-\mathrm{C} 13$ & $1.416(9)$ \\
\hline $\mathrm{C} 5-\mathrm{C} 6$ & $1.406(9)$ & $\mathrm{C} 13-\mathrm{H} 13$ & 0.9300 \\
\hline $\mathrm{C} 5-\mathrm{H} 5$ & 0.9300 & $\mathrm{C} 14-\mathrm{C} 14^{\mathrm{i}}$ & $1.301(13)$ \\
\hline $\mathrm{C} 13-\mathrm{N} 1-\mathrm{C} 1$ & $117.3(6)$ & $\mathrm{C} 9-\mathrm{C} 8-\mathrm{C} 7$ & $120.1(7)$ \\
\hline $\mathrm{N} 1-\mathrm{C} 1-\mathrm{C} 2$ & $117.1(6)$ & $\mathrm{C} 9-\mathrm{C} 8-\mathrm{H} 8$ & 120.0 \\
\hline $\mathrm{N} 1-\mathrm{C} 1-\mathrm{C} 6$ & $122.8(6)$ & $\mathrm{C} 7-\mathrm{C} 8-\mathrm{H} 8$ & 120.0 \\
\hline $\mathrm{C} 2-\mathrm{C} 1-\mathrm{C} 6$ & $120.1(6)$ & $\mathrm{C} 8-\mathrm{C} 9-\mathrm{C} 10$ & $122.1(7)$ \\
\hline $\mathrm{C} 3-\mathrm{C} 2-\mathrm{C} 1$ & $120.8(7)$ & $\mathrm{C} 8-\mathrm{C} 9-\mathrm{H} 9$ & 119.0 \\
\hline $\mathrm{C} 3-\mathrm{C} 2-\mathrm{H} 2$ & 119.6 & $\mathrm{C} 10-\mathrm{C} 9-\mathrm{H} 9$ & 119.0 \\
\hline $\mathrm{C} 1-\mathrm{C} 2-\mathrm{H} 2$ & 119.6 & $\mathrm{C} 11-\mathrm{C} 10-\mathrm{C} 9$ & $119.0(7)$ \\
\hline $\mathrm{C} 2-\mathrm{C} 3-\mathrm{C} 4$ & $119.4(7)$ & $\mathrm{C} 11-\mathrm{C} 10-\mathrm{H} 10$ & 120.5 \\
\hline $\mathrm{C} 2-\mathrm{C} 3-\mathrm{H} 3$ & 120.3 & $\mathrm{C} 9-\mathrm{C} 10-\mathrm{H} 10$ & 120.5 \\
\hline $\mathrm{C} 4-\mathrm{C} 3-\mathrm{H} 3$ & 120.3 & $\mathrm{C} 10-\mathrm{C} 11-\mathrm{C} 12$ & $120.4(7)$ \\
\hline $\mathrm{C} 5-\mathrm{C} 4-\mathrm{C} 3$ & $120.8(7)$ & $\mathrm{C} 10-\mathrm{C} 11-\mathrm{H} 11$ & 119.8 \\
\hline $\mathrm{C} 5-\mathrm{C} 4-\mathrm{H} 4$ & 119.6 & $\mathrm{C} 12-\mathrm{C} 11-\mathrm{H} 11$ & 119.8 \\
\hline $\mathrm{C} 3-\mathrm{C} 4-\mathrm{H} 4$ & 119.6 & $\mathrm{C} 7-\mathrm{C} 12-\mathrm{C} 11$ & $120.1(6)$ \\
\hline $\mathrm{C} 4-\mathrm{C} 5-\mathrm{C} 6$ & $121.2(7)$ & $\mathrm{C} 7-\mathrm{C} 12-\mathrm{C} 13$ & $118.4(6)$ \\
\hline $\mathrm{C} 4-\mathrm{C} 5-\mathrm{H} 5$ & 119.4 & $\mathrm{C} 11-\mathrm{C} 12-\mathrm{C} 13$ & $121.6(6)$ \\
\hline $\mathrm{C} 6-\mathrm{C} 5-\mathrm{H} 5$ & 119.4 & $\mathrm{~N} 1-\mathrm{C} 13-\mathrm{C} 12$ & $125.7(6)$ \\
\hline $\mathrm{C} 1-\mathrm{C} 6-\mathrm{C} 5$ & $117.7(6)$ & $\mathrm{N} 1-\mathrm{C} 13-\mathrm{H} 13$ & 117.1 \\
\hline $\mathrm{C} 1-\mathrm{C} 6-\mathrm{C} 7$ & $118.1(6)$ & $\mathrm{C} 12-\mathrm{C} 13-\mathrm{H} 13$ & 117.1 \\
\hline $\mathrm{C} 5-\mathrm{C} 6-\mathrm{C} 7$ & $124.2(6)$ & $\mathrm{C} 14-\mathrm{C} 14-\mathrm{I} 2$ & $121.2(7)$ \\
\hline $\mathrm{C} 12-\mathrm{C} 7-\mathrm{C} 8$ & $118.3(7)$ & $\mathrm{C} 14^{\mathrm{i}}-\mathrm{C} 14-\mathrm{I} 1$ & $126.2(7)$ \\
\hline $\mathrm{C} 12-\mathrm{C} 7-\mathrm{C} 6$ & $117.7(6)$ & $\mathrm{I} 2-\mathrm{C} 14-\mathrm{I} 1$ & $112.5(3)$ \\
\hline $\mathrm{C} 8-\mathrm{C} 7-\mathrm{C} 6$ & $124.0(6)$ & & \\
\hline
\end{tabular}




$\begin{array}{ll}\mathrm{C} 13-\mathrm{N} 1-\mathrm{C} 1-\mathrm{C} 2 & 179.7(6) \\ \mathrm{C} 13-\mathrm{N} 1-\mathrm{C} 1-\mathrm{C} 6 & 0.2(10) \\ \mathrm{N} 1-\mathrm{C} 1-\mathrm{C} 2-\mathrm{C} 3 & 178.4(7) \\ \mathrm{C} 6-\mathrm{C} 1-\mathrm{C} 2-\mathrm{C} 3 & -2.1(11) \\ \mathrm{C} 1-\mathrm{C} 2-\mathrm{C} 3-\mathrm{C} 4 & 1.8(12) \\ \mathrm{C} 2-\mathrm{C} 3-\mathrm{C} 4-\mathrm{C} 5 & -1.0(12) \\ \mathrm{C} 3-\mathrm{C} 4-\mathrm{C} 5-\mathrm{C} 6 & 0.5(12) \\ \mathrm{N} 1-\mathrm{C} 1-\mathrm{C} 6-\mathrm{C} 5 & -179.0(6) \\ \mathrm{C} 2-\mathrm{C} 1-\mathrm{C} 6-\mathrm{C} 5 & 1.5(10) \\ \mathrm{N} 1-\mathrm{C} 1-\mathrm{C} 6-\mathrm{C} 7 & 1.0(10) \\ \mathrm{C} 2-\mathrm{C} 1-\mathrm{C} 6-\mathrm{C} 7 & -178.5(6) \\ \mathrm{C} 4-\mathrm{C} 5-\mathrm{C} 6-\mathrm{C} 1 & -0.7(10) \\ \mathrm{C} 4-\mathrm{C} 5-\mathrm{C} 6-\mathrm{C} 7 & 179.3(7) \\ \mathrm{C} 1-\mathrm{C} 6-\mathrm{C} 7-\mathrm{C} 12 & -0.9(9) \\ \mathrm{C} 5-\mathrm{C} 6-\mathrm{C} 7-\mathrm{C} 12 & 179.1(7) \\ \mathrm{C} 1-\mathrm{C} 6-\mathrm{C} 7-\mathrm{C} 8 & 179.7(6)\end{array}$

$$
\begin{aligned}
& \mathrm{C} 5-\mathrm{C} 6-\mathrm{C} 7-\mathrm{C} 8 \\
& \mathrm{C} 12-\mathrm{C} 7-\mathrm{C} 8-\mathrm{C} 9 \\
& \mathrm{C} 6-\mathrm{C} 7-\mathrm{C} 8-\mathrm{C} 9 \\
& \mathrm{C} 7-\mathrm{C} 8-\mathrm{C} 9-\mathrm{C} 10 \\
& \mathrm{C} 8-\mathrm{C} 9-\mathrm{C} 10-\mathrm{C} 11 \\
& \mathrm{C} 9-\mathrm{C} 10-\mathrm{C} 11-\mathrm{C} 12 \\
& \mathrm{C} 8-\mathrm{C} 7-\mathrm{C} 12-\mathrm{C} 11 \\
& \mathrm{C} 6-\mathrm{C} 7-\mathrm{C} 12-\mathrm{C} 11 \\
& \mathrm{C} 8-\mathrm{C} 7-\mathrm{C} 12-\mathrm{C} 13 \\
& \mathrm{C} 6-\mathrm{C} 7-\mathrm{C} 12-\mathrm{C} 13 \\
& \mathrm{C} 10-\mathrm{C} 11-\mathrm{C} 12-\mathrm{C} 7 \\
& \mathrm{C} 10-\mathrm{C} 11-\mathrm{C} 12-\mathrm{C} 13 \\
& \mathrm{C} 1-\mathrm{N} 1-\mathrm{C} 13-\mathrm{C} 12 \\
& \mathrm{C} 7-\mathrm{C} 12-\mathrm{C} 13-\mathrm{N} 1 \\
& \mathrm{C} 11-\mathrm{C} 12-\mathrm{C} 13-\mathrm{N} 1
\end{aligned}
$$$$
-0.5(10)
$$$$
-179.9(6)
$$$$
179.3(6)
$$$$
-0.2(10)
$$$$
0.0(10)
$$

Symmetry code: (i) $-x+3 / 2,-y+3 / 2,-z+1$.

Tetraiodoethylene-benzo[f]quinoline (1/2) (2)

Crystal data

$0.5 \mathrm{C}_{2} \mathrm{I}_{4} \cdot \mathrm{C}_{13} \mathrm{H}_{9} \mathrm{~N}$

$M_{r}=445.02$

Triclinic, $P \overline{1}$

$a=7.3179$ (4) $\AA$

$b=8.1089(5) \AA$

$c=11.3252(7) \AA$

$\alpha=97.050(2)^{\circ}$

$\beta=92.059(2)^{\circ}$

$\gamma=95.579(2)^{\circ}$

$V=663.02(7) \AA^{3}$

Data collection

Bruker APEXII CCD

diffractometer

Radiation source: sealed tube

$\varphi$ and $\omega$ scans

Absorption correction: multi-scan

(SADABS; Bruker, 2012)

$T_{\text {min }}=0.638, T_{\max }=0.745$

8567 measured reflections

\section{Refinement}

Refinement on $F^{2}$

Least-squares matrix: full

$R\left[F^{2}>2 \sigma\left(F^{2}\right)\right]=0.028$

$w R\left(F^{2}\right)=0.058$

$S=1.08$

2701 reflections

154 parameters

0 restraints
$Z=2$

$F(000)=412$

$D_{\mathrm{x}}=2.229 \mathrm{Mg} \mathrm{m}^{-3}$

Mo $K \alpha$ radiation, $\lambda=0.71073 \AA$

Cell parameters from 4699 reflections

$\theta=2.8-26.4^{\circ}$

$\mu=4.72 \mathrm{~mm}^{-1}$

$T=296 \mathrm{~K}$

Block, yellow

$0.35 \times 0.32 \times 0.30 \mathrm{~mm}$

2701 independent reflections

2099 reflections with $I>2 \sigma(I)$

$R_{\text {int }}=0.026$

$\theta_{\text {max }}=26.4^{\circ}, \theta_{\min }=2.5^{\circ}$

$h=-9 \rightarrow 9$

$k=-10 \rightarrow 10$

$l=-14 \rightarrow 14$

Hydrogen site location: inferred from neighbouring sites

$\mathrm{H}$-atom parameters constrained

$w=1 /\left[\sigma^{2}\left(F_{\mathrm{o}}^{2}\right)+(0.0189 P)^{2}+0.7413 P\right]$

where $P=\left(F_{\mathrm{o}}^{2}+2 F_{\mathrm{c}}{ }^{2}\right) / 3$

$(\Delta / \sigma)_{\max }=0.001$

$\Delta \rho_{\max }=0.80 \mathrm{e} \AA^{-3}$

$\Delta \rho_{\min }=-0.74$ e $\AA^{-3}$ 


\section{Special details}

Geometry. All esds (except the esd in the dihedral angle between two 1.s. planes) are estimated using the full covariance matrix. The cell esds are taken into account individually in the estimation of esds in distances, angles and torsion angles; correlations between esds in cell parameters are only used when they are defined by crystal symmetry. An approximate (isotropic) treatment of cell esds is used for estimating esds involving l.s. planes.

Fractional atomic coordinates and isotropic or equivalent isotropic displacement parameters $\left(\AA^{2}\right)$

\begin{tabular}{lllll}
\hline & $x$ & $y$ & $z$ & $U_{\text {iso }} / U_{\mathrm{eq}}$ \\
\hline $\mathrm{I} 1$ & $0.20988(4)$ & $0.45307(3)$ & $0.82595(2)$ & $0.04767(10)$ \\
$\mathrm{I} 2$ & $-0.08934(4)$ & $0.75374(3)$ & $0.91391(3)$ & $0.05167(11)$ \\
$\mathrm{N} 1$ & $0.4565(5)$ & $0.3596(5)$ & $0.6400(3)$ & $0.0518(9)$ \\
$\mathrm{C} 1$ & $0.6267(7)$ & $0.4270(6)$ & $0.6615(4)$ & $0.0597(13)$ \\
$\mathrm{H} 1$ & 0.655445 & 0.495377 & 0.733212 & $0.072^{*}$ \\
$\mathrm{C} 2$ & $0.7655(7)$ & $0.4022(6)$ & $0.5841(5)$ & $0.0613(13)$ \\
$\mathrm{H} 2$ & 0.883874 & 0.453358 & 0.603495 & $0.074^{*}$ \\
C3 & $0.7274(6)$ & $0.3026(6)$ & $0.4794(5)$ & $0.0541(12)$ \\
$\mathrm{H} 3$ & 0.819507 & 0.286190 & 0.425958 & $0.065^{*}$ \\
C4 & $0.5492(5)$ & $0.2240(5)$ & $0.4514(4)$ & $0.0399(10)$ \\
C5 & $0.4171(5)$ & $0.2587(5)$ & $0.5350(4)$ & $0.0389(9)$ \\
C6 & $0.2327(6)$ & $0.1869(5)$ & $0.5119(4)$ & $0.0470(11)$ \\
H6 & 0.144788 & 0.211154 & 0.566982 & $0.056^{*}$ \\
C7 & $0.1844(6)$ & $0.0845(5)$ & $0.4114(4)$ & $0.0471(11)$ \\
H7 & 0.062732 & 0.038787 & 0.398340 & $0.057^{*}$ \\
C8 & $0.3128(6)$ & $0.0431(5)$ & $0.3238(4)$ & $0.0424(10)$ \\
C9 & $0.4960(6)$ & $0.1144(5)$ & $0.3424(4)$ & $0.0418(10)$ \\
C10 & $0.6199(7)$ & $0.0731(6)$ & $0.2544(4)$ & $0.0561(12)$ \\
H10 & 0.741510 & 0.119847 & 0.264207 & $0.067^{*}$ \\
C11 & $0.5643(9)$ & $-0.0348(7)$ & $0.1546(5)$ & $0.0730(16)$ \\
H11 & 0.648615 & -0.060734 & 0.097457 & $0.088^{*}$ \\
C12 & $0.3842(9)$ & $-0.1063(7)$ & $0.1373(4)$ & $0.0716(16)$ \\
H12 & 0.347907 & -0.180218 & 0.069304 & $0.086^{*}$ \\
C13 & $0.2612(7)$ & $-0.0676(6)$ & $0.2203(4)$ & $0.0555(12)$ \\
H13 & 0.140145 & -0.115380 & 0.208420 & $0.067^{*}$ \\
C14 & $0.0238(6)$ & $0.5360(5)$ & $0.9536(4)$ & $0.0449(10)$ \\
& & & & \\
\hline
\end{tabular}

Atomic displacement parameters $\left(\AA^{2}\right)$

\begin{tabular}{lllllll}
\hline & $U^{11}$ & $U^{22}$ & $U^{33}$ & $U^{12}$ & $U^{13}$ & $U^{23}$ \\
\hline $\mathrm{I} 1$ & $0.0579(2)$ & $0.04585(18)$ & $0.04064(16)$ & $0.01385(14)$ & $0.01030(14)$ & $0.00193(12)$ \\
$\mathrm{I} 2$ & $0.0700(2)$ & $0.04158(18)$ & $0.04756(18)$ & $0.02041(15)$ & $0.00980(15)$ & $0.00849(13)$ \\
$\mathrm{N} 1$ & $0.058(3)$ & $0.051(2)$ & $0.049(2)$ & $0.0117(19)$ & $0.003(2)$ & $0.0083(18)$ \\
$\mathrm{C} 1$ & $0.068(3)$ & $0.052(3)$ & $0.058(3)$ & $0.007(3)$ & $-0.016(3)$ & $0.006(2)$ \\
$\mathrm{C} 2$ & $0.042(3)$ & $0.067(3)$ & $0.076(4)$ & $-0.002(2)$ & $-0.010(3)$ & $0.024(3)$ \\
$\mathrm{C} 3$ & $0.039(3)$ & $0.055(3)$ & $0.073(3)$ & $0.006(2)$ & $0.013(2)$ & $0.026(3)$ \\
$\mathrm{C} 4$ & $0.036(2)$ & $0.041(2)$ & $0.049(2)$ & $0.0109(18)$ & $0.008(2)$ & $0.0221(19)$ \\
$\mathrm{C} 5$ & $0.041(2)$ & $0.036(2)$ & $0.043(2)$ & $0.0108(18)$ & $0.0065(19)$ & $0.0135(18)$ \\
$\mathrm{C} 6$ & $0.042(2)$ & $0.054(3)$ & $0.048(3)$ & $0.008(2)$ & $0.015(2)$ & $0.009(2)$
\end{tabular}


supporting information

\begin{tabular}{|c|c|c|c|c|c|c|}
\hline $\mathrm{C} 7$ & $0.036(2)$ & $0.051(3)$ & $0.056(3)$ & $0.003(2)$ & $0.006(2)$ & $0.016(2)$ \\
\hline $\mathrm{C} 8$ & $0.051(3)$ & $0.036(2)$ & $0.044(2)$ & $0.0087(19)$ & $0.006(2)$ & $0.0144(18)$ \\
\hline C9 & $0.046(2)$ & $0.042(2)$ & $0.044(2)$ & 0.0161 (19) & $0.014(2)$ & $0.0199(19)$ \\
\hline $\mathrm{C} 10$ & 0.059 (3) & $0.066(3)$ & $0.052(3)$ & $0.023(2)$ & $0.021(2)$ & $0.022(2)$ \\
\hline C11 & $0.099(5)$ & $0.080(4)$ & $0.051(3)$ & $0.043(4)$ & $0.031(3)$ & $0.018(3)$ \\
\hline $\mathrm{C} 12$ & $0.115(5)$ & $0.059(3)$ & $0.044(3)$ & $0.025(3)$ & $0.007(3)$ & $0.004(2)$ \\
\hline $\mathrm{C} 13$ & $0.072(3)$ & $0.047(3)$ & 0.049 (3) & $0.009(2)$ & $-0.003(3)$ & $0.010(2)$ \\
\hline $\mathrm{C} 14$ & $0.054(3)$ & $0.039(2)$ & $0.041(2)$ & $0.011(2)$ & $0.002(2)$ & $-0.0017(18)$ \\
\hline
\end{tabular}

Geometric parameters $\left(\AA,{ }^{\circ}\right)$

\begin{tabular}{|c|c|c|c|}
\hline $\mathrm{I} 1-\mathrm{C} 14$ & $2.116(4)$ & $\mathrm{C} 6-\mathrm{H} 6$ & 0.9300 \\
\hline $\mathrm{I} 2-\mathrm{C} 14$ & $2.111(4)$ & $\mathrm{C} 7-\mathrm{C} 8$ & $1.423(6)$ \\
\hline $\mathrm{N} 1-\mathrm{C} 1$ & $1.313(6)$ & $\mathrm{C} 7-\mathrm{H} 7$ & 0.9300 \\
\hline $\mathrm{N} 1-\mathrm{C} 5$ & $1.363(5)$ & $\mathrm{C} 8-\mathrm{C} 13$ & $1.402(6)$ \\
\hline $\mathrm{C} 1-\mathrm{C} 2$ & $1.379(7)$ & $\mathrm{C} 8-\mathrm{C} 9$ & $1.405(6)$ \\
\hline $\mathrm{C} 1-\mathrm{H} 1$ & 0.9300 & $\mathrm{C} 9-\mathrm{C} 10$ & $1.403(6)$ \\
\hline $\mathrm{C} 2-\mathrm{C} 3$ & $1.354(7)$ & $\mathrm{C} 10-\mathrm{C} 11$ & $1.365(7)$ \\
\hline $\mathrm{C} 2-\mathrm{H} 2$ & 0.9300 & $\mathrm{C} 10-\mathrm{H} 10$ & 0.9300 \\
\hline $\mathrm{C} 3-\mathrm{C} 4$ & $1.402(6)$ & $\mathrm{C} 11-\mathrm{C} 12$ & $1.384(8)$ \\
\hline $\mathrm{C} 3-\mathrm{H} 3$ & 0.9300 & $\mathrm{C} 11-\mathrm{H} 11$ & 0.9300 \\
\hline $\mathrm{C} 4-\mathrm{C} 5$ & $1.401(5)$ & $\mathrm{C} 12-\mathrm{C} 13$ & $1.355(7)$ \\
\hline $\mathrm{C} 4-\mathrm{C} 9$ & $1.448(6)$ & $\mathrm{C} 12-\mathrm{H} 12$ & 0.9300 \\
\hline $\mathrm{C} 5-\mathrm{C} 6$ & $1.418(6)$ & $\mathrm{C} 13-\mathrm{H} 13$ & 0.9300 \\
\hline $\mathrm{C} 6-\mathrm{C} 7$ & $1.339(6)$ & $\mathrm{C} 14-\mathrm{C} 14^{\mathrm{i}}$ & $1.305(8)$ \\
\hline $\mathrm{C} 1-\mathrm{N} 1-\mathrm{C} 5$ & $117.4(4)$ & $\mathrm{C} 13-\mathrm{C} 8-\mathrm{C} 9$ & $119.4(4)$ \\
\hline $\mathrm{N} 1-\mathrm{C} 1-\mathrm{C} 2$ & $123.8(5)$ & $\mathrm{C} 13-\mathrm{C} 8-\mathrm{C} 7$ & $121.7(4)$ \\
\hline $\mathrm{N} 1-\mathrm{C} 1-\mathrm{H} 1$ & 118.1 & $\mathrm{C} 9-\mathrm{C} 8-\mathrm{C} 7$ & $118.9(4)$ \\
\hline $\mathrm{C} 2-\mathrm{C} 1-\mathrm{H} 1$ & 118.1 & $\mathrm{C} 10-\mathrm{C} 9-\mathrm{C} 8$ & $118.0(4)$ \\
\hline $\mathrm{C} 3-\mathrm{C} 2-\mathrm{C} 1$ & $119.2(4)$ & $\mathrm{C} 10-\mathrm{C} 9-\mathrm{C} 4$ & $123.0(4)$ \\
\hline $\mathrm{C} 3-\mathrm{C} 2-\mathrm{H} 2$ & 120.4 & $\mathrm{C} 8-\mathrm{C} 9-\mathrm{C} 4$ & $119.0(4)$ \\
\hline $\mathrm{C} 1-\mathrm{C} 2-\mathrm{H} 2$ & 120.4 & $\mathrm{C} 11-\mathrm{C} 10-\mathrm{C} 9$ & $120.9(5)$ \\
\hline $\mathrm{C} 2-\mathrm{C} 3-\mathrm{C} 4$ & $120.1(4)$ & $\mathrm{C} 11-\mathrm{C} 10-\mathrm{H} 10$ & 119.6 \\
\hline $\mathrm{C} 2-\mathrm{C} 3-\mathrm{H} 3$ & 120.0 & $\mathrm{C} 9-\mathrm{C} 10-\mathrm{H} 10$ & 119.6 \\
\hline $\mathrm{C} 4-\mathrm{C} 3-\mathrm{H} 3$ & 120.0 & $\mathrm{C} 10-\mathrm{C} 11-\mathrm{C} 12$ & $121.0(5)$ \\
\hline $\mathrm{C} 3-\mathrm{C} 4-\mathrm{C} 5$ & $116.5(4)$ & $\mathrm{C} 10-\mathrm{C} 11-\mathrm{H} 11$ & 119.5 \\
\hline $\mathrm{C} 3-\mathrm{C} 4-\mathrm{C} 9$ & $124.0(4)$ & $\mathrm{C} 12-\mathrm{C} 11-\mathrm{H} 11$ & 119.5 \\
\hline $\mathrm{C} 5-\mathrm{C} 4-\mathrm{C} 9$ & $119.5(4)$ & $\mathrm{C} 13-\mathrm{C} 12-\mathrm{C} 11$ & $119.5(5)$ \\
\hline $\mathrm{N} 1-\mathrm{C} 5-\mathrm{C} 4$ & $123.0(4)$ & $\mathrm{C} 13-\mathrm{C} 12-\mathrm{H} 12$ & 120.3 \\
\hline $\mathrm{N} 1-\mathrm{C} 5-\mathrm{C} 6$ & $117.1(4)$ & $\mathrm{C} 11-\mathrm{C} 12-\mathrm{H} 12$ & 120.3 \\
\hline $\mathrm{C} 4-\mathrm{C} 5-\mathrm{C} 6$ & $119.9(4)$ & $\mathrm{C} 12-\mathrm{C} 13-\mathrm{C} 8$ & $121.3(5)$ \\
\hline $\mathrm{C} 7-\mathrm{C} 6-\mathrm{C} 5$ & $120.4(4)$ & $\mathrm{C} 12-\mathrm{C} 13-\mathrm{H} 13$ & 119.4 \\
\hline $\mathrm{C} 7-\mathrm{C} 6-\mathrm{H} 6$ & 119.8 & $\mathrm{C} 8-\mathrm{C} 13-\mathrm{H} 13$ & 119.4 \\
\hline $\mathrm{C} 5-\mathrm{C} 6-\mathrm{H} 6$ & 119.8 & $\mathrm{C} 14^{\mathrm{i}}-\mathrm{C} 14-\mathrm{I} 2$ & $121.4(4)$ \\
\hline $\mathrm{C} 6-\mathrm{C} 7-\mathrm{C} 8$ & $122.3(4)$ & $\mathrm{C} 14-\mathrm{C} 14-\mathrm{I} 1$ & $126.2(4)$ \\
\hline $\mathrm{C} 6-\mathrm{C} 7-\mathrm{H} 7$ & 118.9 & $\mathrm{I} 2-\mathrm{C} 14-\mathrm{I} 1$ & $112.33(18)$ \\
\hline $\mathrm{C} 8-\mathrm{C} 7-\mathrm{H} 7$ & 118.9 & & \\
\hline
\end{tabular}




$\begin{array}{llll}\mathrm{C} 5-\mathrm{N} 1-\mathrm{C} 1-\mathrm{C} 2 & 0.5(7) & \mathrm{C} 13-\mathrm{C} 8-\mathrm{C} 9-\mathrm{C} 10 & 1.1(6) \\ \mathrm{N} 1-\mathrm{C} 1-\mathrm{C} 2-\mathrm{C} 3 & -0.3(8) & \mathrm{C} 7-\mathrm{C} 8-\mathrm{C} 9-\mathrm{C} 10 & -179.4(4) \\ \mathrm{C} 1-\mathrm{C} 2-\mathrm{C} 3-\mathrm{C} 4 & -0.9(7) & \mathrm{C} 13-\mathrm{C} 8-\mathrm{C} 9-\mathrm{C} 4 & 1.6(6) \\ \mathrm{C} 2-\mathrm{C} 3-\mathrm{C} 4-\mathrm{C} 5 & 1.7(6) & \mathrm{C} 3-\mathrm{C} 8-\mathrm{C} 9-\mathrm{C} 9-\mathrm{C} 40 & 1.3(6) \\ \mathrm{C} 2-\mathrm{C} 3-\mathrm{C} 4-\mathrm{C} 9 & \mathrm{C} 5-\mathrm{C} 4-\mathrm{C} 9-\mathrm{C} 10 & -179.8(4) \\ \mathrm{C} 1-\mathrm{N} 1-\mathrm{C} 5-\mathrm{C} 4 & \mathrm{C} 3-\mathrm{C} 4-\mathrm{C} 9-\mathrm{C} 8 & -179.7(4) \\ \mathrm{C} 1-\mathrm{N} 1-\mathrm{C} 5-\mathrm{C} 6 & \mathrm{C} 5-\mathrm{C} 4-\mathrm{C} 9-\mathrm{C} 8 & -0.9(6) \\ \mathrm{C} 3-\mathrm{C} 4-\mathrm{C} 5-\mathrm{N} 1 & \mathrm{C} 8-\mathrm{C} 9-\mathrm{C} 10-\mathrm{C} 11 & 178.1(4) \\ \mathrm{C} 9-\mathrm{C} 4-\mathrm{C} 5-\mathrm{N} 1 & \mathrm{C} 4-\mathrm{C} 9-\mathrm{C} 10-\mathrm{C} 11 & 0.1(8) \\ \mathrm{C} 3-\mathrm{C} 4-\mathrm{C} 5-\mathrm{C} 6 & \mathrm{C} 9-\mathrm{C} 10-\mathrm{C} 11-\mathrm{C} 12 & 0.4(8) \\ \mathrm{C} 9-\mathrm{C} 4-\mathrm{C} 5-\mathrm{C} 6 & \mathrm{C} 10-\mathrm{C} 11-\mathrm{C} 12-\mathrm{C} 13 & -0.2(7) \\ \mathrm{N} 1-\mathrm{C} 5-\mathrm{C} 6-\mathrm{C} 7 & \mathrm{C} 11-\mathrm{C} 12-\mathrm{C} 13-\mathrm{C} 8 & -0.5(6) \\ \mathrm{C} 4-\mathrm{C} 5-\mathrm{C} 6-\mathrm{C} 7 & -0.3(6) & \mathrm{C} 9-\mathrm{C} 8-\mathrm{C} 13-\mathrm{C} 12 & 180.0(4) \\ \mathrm{C} 5-\mathrm{C} 6-\mathrm{C} 7-\mathrm{C} 8 & -179.0(4) & \mathrm{C} 7-\mathrm{C} 8-\mathrm{C} 13-\mathrm{C} 12 & \\ \mathrm{C} 6-\mathrm{C} 7-\mathrm{C} 8-\mathrm{C} 13 & 0.8(6) & & \\ \mathrm{C} 6-\mathrm{C} 7-\mathrm{C} 8-\mathrm{C} 9 & -0.1(7) & & \end{array}$

Symmetry code: (i) $-x,-y+1,-z+2$.

Hydrogen-bond geometry $\left(\AA,{ }^{\circ}\right)$

\begin{tabular}{lllll}
\hline$D-\mathrm{H} \cdots A$ & $D-\mathrm{H}$ & $\mathrm{H} \cdots A$ & $D \cdots A$ & $D-\mathrm{H} \cdots A$ \\
\hline $\mathrm{C} 1-\mathrm{H} 1 \cdots \mathrm{I} 2^{\mathrm{ii}}$ & 0.93 & 3.16 & $4.019(5)$ & 155 \\
$\mathrm{C} 6-\mathrm{H} 6 \cdots \mathrm{I} 1$ & 0.93 & 3.31 & $3.945(4)$ & 127 \\
\hline
\end{tabular}

Symmetry code: (ii) $x+1, y, z$. 